\title{
Le devenir de la première union commencée hors mariage : une analyse comparative entre la France, l'Italie, la Suède et la Suisse à partir des enquêtes FFS des années 1990 The Evolution of the First Union Begun Outside Marriage: A Comparative Analysis of France, Italy, Sweden and Switzerland Based on Fertility and Family Surveys of the 1990s
}

\section{Laurence Charton}

Volume 34, numéro 2, automne 2005

Démographie historique

URI : https://id.erudit.org/iderudit/014016ar

DOI : https://doi.org/10.7202/014016ar

Aller au sommaire du numéro

Éditeur(s)

Association des démographes du Québec

ISSN

0380-1721 (imprimé)

1705-1495 (numérique)

Découvrir la revue

Citer cet article

Charton, L. (2005). Le devenir de la première union commencée hors mariage : une analyse comparative entre la France, l'Italie, la Suède et la Suisse à partir des enquêtes FFS des années 1990. Cahiers québécois de démographie, 34(2),

349-374. https://doi.org/10.7202/014016ar

\section{Résumé de l'article}

Aujourd'hui, de plus en plus de couples commencent, en Europe, une première expérience conjugale hors mariage. Le devenir de ces unions fait l'objet de diverses questions : sont-elles plus fragiles que l'union maritale ? Sont-elles des épisodes de vie de couple temporaires? Aboutissent-elles au mariage? Sont-elles une nouvelle forme d'union parallèle à l'institution du mariage ? Existe-t-il différentes formes de cohabitation hors mariage selon les pays? Enfin, quelle est la part de l'environnement familial et social dans le devenir de l'union ? Cet article se propose d'apporter des éléments de réponses à ces questions en utilisant les données recueillies au cours des années 1990 par les enquêtes Fécondité et famille, en France, en Italie, en Suède et en Suisse, pays représentatifs de la variété des situations conjugales et fécondes en Europe occidentale. Les méthodes d'analyses des biographies ont été retenues pour prendre en compte les durées écoulées et les caractéristiques individuelles et contextuelles. 
Cahiers québécois de démographie

Vol. 34, no 2, automne 2005, p. 349-374.

\title{
Le devenir de la première union commencée hors mariage : une analyse comparative entre la France, 1'Italie, la Suède et la Suisse à partir des enquêtes FFS des années 1990
}

\author{
Laurence CHARTON *
}

\begin{abstract}
Aujourd'hui, de plus en plus de couples commencent, en Europe, une première expérience conjugale hors mariage. Le devenir de ces unions fait l'objet de diverses questions : sont-elles plus fragiles que l'union maritale? Sont-elles des épisodes de vie de couple temporaires? Aboutissent-elles au mariage? Sontelles une nouvelle forme d'union parallèle à l'institution du mariage ? Existe-t-il différentes formes de cohabitation hors mariage selon les pays? Enfin, quelle est la part de l'environnement familial et social dans le devenir de l'union? Cet article se propose d'apporter des éléments de réponses à ces questions en utilisant les données recueillies au cours des années 1990 par les enquêtes Fécondité et famille, en France, en Italie, en Suède et en Suisse, pays représentatifs de la variété des situations conjugales et fécondes en Europe occidentale. Les méthodes d'analyses des biographies ont été retenues pour prendre en compte les durées écoulées et les caractéristiques individuelles et contextuelles.
\end{abstract}

English abstract, p. 374.

T a proportion de personnes vivant en couple sans être mariées $\mathcal{L}$ est relativement élevée en Europe, malgré l'existence de grandes différences entre les pays, notamment entre les pays du Nord et du Sud (Roussel, 1992; Kaufmann, 1994; Pinelli et al., 2001). La cohabitation hors mariage s'est répandue d'abord dans les pays scandinaves, puis s'est diffusée dans toute l'Europe au début des années 1980, à l'exception des pays du sud, où elle est toujours peu présente (Trost, 1975; Hoffmann-Nowotny et Fux, 1991). La multiplication de ces unions a eu lieu en même temps que l'augmentation du nombre des divorces, la diminution de la fécondité et la baisse de la nuptialité, conduisant à ce que Van de Kaa (1987) a nommé la " deuxième transition démographique ".

Université Marc Bloch, Strasbourg, France. 
Si cette expression ne fait pas l'unanimité (Hoffmann-Nowotny, 1988; Pavlik, 1989; Cliquet, 1991), la plupart des auteurs s'accordent néanmoins à reconnaître que depuis les années 1965 des changements importants ont touché la famille au niveau des comportements de procréation et des modes de vie en couple (Roussel, 1989; Höhn, 1997), aboutissant à la mise en place d'un nouveau modèle familial.

Diverses interprétations ont été données pour expliquer l'émergence des nouvelles formes d'union dans le paysage conjugal. Pour certains, les unions hors mariage étaient d'abord des " cohabitations juvéniles" (Roussel, 1978) et des "préludes au mariage " (Villeneuve-Gokalp, 1990; Singly, 1992), car elles concernaient en premier lieu des jeunes dont l'entrée dans la vie adulte semblait retardée avec l'allongement de la scolarité et la précarité de l'emploi. Elles se terminaient d'ailleurs le plus souvent par un passage devant l'officier d'état civil. Ces interprétations se fondent notamment sur la théorie économique des attentes déçues d'Easterlin (1976), selon laquelle, dans un contexte où l'évolution économique exige que chacun soit prêt à changer d'emploi et de métier plusieurs fois dans sa vie, à se réorienter et à se former en permanence, l'incertitude que les personnes peuvent ressentir à l'égard de leur vie professionnelle peut se retrouver également au niveau de leur vie personnelle, voire familiale. Schématiquement, les difficultés rencontrées par les nombreuses générations à s'insérer sur le marché du travail inciteraient leurs membres à modifier leur comportement en restant célibataires, en ayant moins d'enfants, en vivant avec leurs parents ou avec d'autres personnes, en formant des unions libres. Le désir de consommation élevé, voire croissant, des générations montantes, alors que la situation économique se détériore, engendrerait aussi un retard dans la formation des familles. La difficulté à s'insérer dans la vie active en fin de formation aurait, de la même manière, une influence importante sur la formation des couples et la constitution des familles (Ponthieux, 1997). Pour Dormont et Dufour-Kippelen (2000), l'acquisition d'une autonomie financière serait la condition sine qua non à une installation autonome, tandis que le premier emploi aurait, selon Ekert-Jaffe et Solaz (2001), plus d'effet sur la première mise en couple que l'inverse.

Le travail des femmes à l'extérieur du foyer est souvent présenté aussi comme variable explicative des transformations conjugales. Selon les théories économistes, celle de Becker (1960, 1981) par exemple, le pouvoir que les femmes acquiè- 
rent avec un salaire fait décliner "le gain du mariage ". Au dire de Becker, les individus se marient quand ce gain l'emporte pour les deux partenaires sur l'avantage de rester célibataire. L'indépendance financière apportée aux femmes par un salaire aurait fait diminuer l'attrait du mariage au profit de la cohabitation hors mariage. Cette théorie a été critiquée, notamment par Oppenheimer (1997), qui a constaté que dans les années 1980, aux États-Unis, les femmes qui avaient un niveau d'instruction plus élevé, c'est-à-dire une meilleure position sur le marché du travail et un revenu plus élevé, avaient tendance à se marier dans des proportions plus fortes. Les nouvelles théories économistes ont depuis incorporé dans la théorie du mariage - à côté des modifications de type purement économique (niveau de vie, consommation espérée) - la confiance réciproque, la compréhension, la reconnaissance etc. C'est la qualité espérée de l'union qui jouerait un rôle central dans les nouvelles théories économistes ou sociales de l'échange (England et Farkas, 1986). Alors que les économistes néoclassiques envisagent par ailleurs une montée du célibat en raison d'un moindre rendement du mariage pour les femmes, Oppenheimer (1988) postule plutôt que le retard au mariage serait dû à l'élévation du niveau d'instruction des femmes, qui aurait également accru leurs exigences minimales à l'égard de leur futur conjoint. L'allongement de la phase prémaritale résulterait alors d'une "recherche " de conjoint plus attentive et de l'introduction d'une période d'essai. C'est aussi ce que soutiennent Lesthaeghe (1983) et Rindfuss et Vanden Heuvel (1990).

Les statistiques officielles ne permettent pas de décrire de manière détaillée les processus de formation du couple et de la famille en Europe, ni de cerner l'impact des facteurs individuels et contextuels sur la forme d'union choisie et sur son devenir. Par contre, les données issues d'enquêtes rétrospectives récentes en donnent quelques indications. Elles montrent notamment un écart important entre pays dans le niveau de cohabitation hors mariage (Klijzing et Macura, 1996; Charton et Duchêne, 2002) et permettent de tenter de répondre à certaines questions.

Par exemple, ces unions sont-elles plus fragiles que les unions maritales ? Sont-elles des épisodes de vie de couple temporaires ? Aboutissent-elles au mariage ? Sont-elles une nouvelle forme d'union parallèle à l'institution du mariage ? Existe-il différentes formes de cohabitation hors mariage selon les pays ? Enfin, quelle est la part de l'environnement familial et social dans le devenir de l'union? 
Seule une approche dynamique et comparative peut nous aider à mieux comprendre les différences de comportements conjugaux et les facteurs déterminants dans leur évolution.

\section{SOURCE DE DONNÉES, MÉTHODES D'ANALYSE ET CHOIX DES PAYS}

Au début des années 1990, la Commission économique pour l'Europe (CEE) des Nations Unies a lancé un vaste projet d'enquêtes comparatives sur la famille et la fécondité, dites FFS (Fertility and Family Surveys), dans 24 pays développés économiquement. Chaque pays participant au projet a réalisé une enquête à partir d'un questionnaire standardisé, auprès de deux échantillons indépendants d'hommes (minimum 2000 personnes) et de femmes (minimum 3000 personnes) représentatifs de sa population résidante. L'objectif de ces enquêtes était d'observer et d'analyser les biographies familiales et professionnelles des adultes (20-49 ans). Des informations détaillées ont ainsi été recueillies sur des périodes successives - et dans certains cas simultanées - de la vie de couple, sur la forme de ces unions, la naissance des enfants, les naissances souhaitées, les pratiques contraceptives ou encore les parcours scolaires et professionnels des personnes interrogées (ECE, 1989).

Pour mesurer le rôle des facteurs familiaux, contextuels et individuels sur le devenir de l'union non maritale, trois analyses ont été réalisées à partir d'un modèle semi-paramétrique de Cox (1972). Il s'agissait ici de s'intéresser aux facteurs susceptibles d'accélérer ou de freiner la sortie de l'union non maritale, soit par une séparation, soit par un mariage. À chaque âge, les probabilités de passer de l'état de cohabitant à l'état de marié (par exemple) sont obtenues en divisant le nombre d'événements observés (le mariage) par le nombre de personnes n'ayant pas encore vécu l'événement (la séparation est considérée comme un phénomène perturbateur entraînant la sortie du champ d'observation). À partir de ces probabilités de transition, des quotients instantanés ont été calculés, représentant la probabilité de se marier au cours d'un intervalle de temps minime. Le modèle de Cox peut être exprimé par l'équation $h(t, Z)=h O(t) * \exp \left(Z^{\prime} \beta\right)$, où $h(t, Z)$ représente la fonction du quotient instantané de se marier au cours du temps $t$ des individus en couple hors mariage, dont les caractéristiques individuelles, contextuelles et familiales sont définies par le vecteur $Z$. Le terme $h_{0}(t)$ représente, pour un individu de référence, le "risque de base " de passer de l'état de 
cohabitant à celui de marié. Ce risque est influencé par les différentes caractéristiques individuelles, contextuelles ou familiales, qui dans le modèle peuvent être traduites par $\exp \left(Z^{\prime} \beta\right)$. Ainsi, $\exp \left(Z^{\prime} \beta\right)=\exp \left(Z_{1}, \beta_{1+} Z_{2}, \beta_{2}+Z_{2}, \beta_{2+}\right)$ représente le vecteur $Z$ de ces caractéristiques, multiplié par le vecteur $\beta$ des coefficients de proportionnalité associés à chacune d'elles, dont certaines sont des covariables dépendantes du temps (Blossfeld et al., 1989; Blossfeld et Rohwer, 1995).

Le modèle de Cox a donc pour objectif de mesurer le risque statistique de se marier en fonction du temps écoulé depuis la mise en couple hors mariage. Le fait que certaines personnes aient vécu plusieurs années en couple tandis que d'autres n'ont été observées que pour quelques mois est pris en compte au moment de l'estimation des paramètres du modèle. Une hypothèse du modèle de Cox est celle de la proportionnalité des risques. Autrement dit, les échelles de risque des différentes souspopulations définies par les modalités des variables sont supposées évoluer de manière proportionnelle, quel que soit le temps écoulé depuis l'événement d'origine. Cette hypothèse a été testée par différentes analyses de survie non paramétriques. Bien qu'il ne soit pas possible d'exclure strictement une absence de proportionnalité, les résultats obtenus par les analyses non paramétriques paraissent légitimer l'utilisation du modèle de Cox pour ces analyses.

Trois modèles semi-paramétriques ont ainsi été construits pour chaque sexe et pour chaque pays. Le premier s'intéresse à la survie dans l'état de cohabitant non marié, quel que soit le type de sortie (mariage ou séparation). Le deuxième modèle a été appliqué à la sortie par un mariage. Dans ce cas, la séparation est considérée comme un phénomène concurrentiel ou alternatif, entraînant la sortie de la population des cohabitants. Le troisième modèle testé s'intéresse au phénomène de la séparation. Dans ce cas, le mariage est considéré comme un phénomène entrant en concurrence (risque concurrent).

La France, l'Italie, la Suède et la Suisse sont les quatre pays retenus pour nos analyses. Ces pays sont en effet révélateurs de certains comportements familiaux dans différents espaces géographiques européens, et des différences dans l'évolution des comportements conjugaux et féconds ont pu y être observées. L'objectif est, en effet, d'examiner aussi bien la diversité des comportements et des processus familiaux que leurs évolutions. Ainsi, la Suède a été retenue en tant que pays précurseur, depuis les années 1960, des changements familiaux. 
L'Italie est le pays où les comportements familiaux paraissent toujours s'inscrire dans un cadre institutionnel. La Suisse est le seul pays en Europe où, malgré un niveau de cohabitation hors mariage important, les naissances hors institution matrimoniale restent rares. La France est le pays où les comportements familiaux sont proches de ceux de la Suède, dans un contexte culturel différent. Il sera notamment question d'observer dans ces deux pays si l'évolution des comportements familiaux s'est faite de manière identique ou selon des processus distincts.

Les enquêtes FFS ont été réalisées dans chacun de ces pays au cours d'une période relativement proche (entre 1992 et 1996), auprès de femmes et d'hommes appartenant à des générations semblables. Si, en France, en Italie et en Suisse, des groupes d'âge quinquennaux ont pu être constitués en rassemblant cinq générations successives, cela n'a pas pu être le cas en Suède, où seulement trois générations masculines et cinq générations féminines ont été interrogées. Dans les tableaux présentés il faudra ainsi, pour la Suède, faire correspondre

- au groupe d'âge 20-24 ans : 23 ans;

- au groupe d'âge 25-29 ans : 28 ans;

- au groupe d'âge 30-34 ans : 32 ans;

- au groupe d'âge 35-39 ans : 38 ans, et

- au groupe d'âge 40-44 ans : 42 ans.

Enfin, chacun de ces quatre pays se retrouve dans un groupe de pays d'une typologie (Roussel, 1992) reflétant à la fin des années 1980 , dans 15 pays d'Europe occidentale, la variété des situations en matière de fécondité et de conjugalité.

\section{VIVRE EN COUPLE HORS MARIAGE EN FRANCE, EN ITALIE, EN SUÈDE ET EN SUISSE}

En France, en Suède et en Suisse, la plupart des personnes arrivant aux âges reproductifs commencent aujourd'hui leur vie conjugale dans le cadre d'une cohabitation hors mariage. En Italie, bien que cette forme d'union reste toujours minoritaire, un certain nombre de couples optent néanmoins, pendant un temps plus ou moins long, pour cette nouvelle forme de cohabitation conjugale (Charton, 2003).

Ainsi, les unions hors mariage sont de durée relativement courte avant d'aboutir à un mariage ou, plus rarement (sauf pour les hommes en Italie), à une séparation. Plus précisé- 
ment, au moment des enquêtes FFS, parmi les personnes qui ont terminé leur première expérience conjugale hors mariage, près de sept hommes et femmes sur dix en France (respectivement 67 pour cent et 71 pour cent) et en Suisse (respectivement 71 pour cent et 72 pour cent) ont officialisé leur première union. En Suède, ces proportions atteignent respectivement 56 pour cent et 61 pour cent, tandis qu'en Italie 47 pour cent des hommes qui ont commencé une première expérience conjugale hors mariage se sont mariés (plus de la moitié se sont donc séparés de leur partenaire), tout comme 79 pour cent des femmes.

Villeneuve-Gokalp (1990) a élaboré une classification des unions non maritales en fonction des événements survenus au cours des trois premières années ayant suivi la mise en union. Huit situations ont été observées, à la date des enquêtes, entre le moment de la mise en couple et les trois années qui ont suivi celle-ci : 1) un mariage direct; 2) une union suivie d'un mariage; 3) une union suivie d'une naissance; 4) une union hors mariage qui s'installe dans la durée (sans mariage, ni naissance); 5) une union rompue dans l'année de la mise en couple; 6) une union rompue après 12 à 36 mois de cohabitation; 7) une situation de " sans union " à l'enquête; et 8) une situation d'union " tronquée " (union observée moins de trois ans). En adaptant cette classification (voir le tableau A, en annexe), on observe tout d'abord qu'en Suède, dès la cohorte d'âge la plus élevée (43 ans), près de huit femmes et hommes sur dix (respectivement 78 pour cent et 82 pour cent) ont cohabité hors des liens du mariage. Ce mode de vie conjugale constituait dans un premier temps une nouvelle étape du processus matrimonial, puisque environ 55 pour cent des femmes des cohortes d'âge 43 ans et 38 ans et près de 58 pour cent des hommes de 43 ans ont officialisé leur union dans les trois années qui ont suivi leur mise en ménage. Près de 5 pour cent des femmes de 43 ans et 10 pour cent des hommes de la même cohorte d'âge ont par ailleurs vécu en couple, sans enfant, durant trois années au moins, tandis qu'environ 17 pour cent des femmes et 14 pour cent des hommes ont eu un enfant en couple non marié.

Au fil des cohortes d'âge, le nombre de mariages " directs " et de cohabitations prénuptiales parmi l'ensemble des premières unions n'a ensuite cessé de diminuer, atteignant à partir de la cohorte d'âge 28 ans un niveau égal (pour les femmes) ou inférieur (pour les hommes) à celui des unions non rompues et n'ayant pas abouti à un mariage. Environ 34 pour cent des femmes de 28 ans se sont mariées dans les trois années ayant 
suivi la mise en union avec leur premier conjoint, tandis que 42 pour cent ne l'ont pas fait ( 22 pour cent vivent en union hors mariage féconde et 13 pour cent en union stable, et 6 pour cent se sont séparées). De même, si 23 pour cent des hommes se sont mariés dans les trois années, 44 pour cent d'entre eux sont restés célibataires ( 20 pour cent vivent en union libre et 14 pour cent en union stable, et 10 pour cent se sont séparés). La cohabitation hors mariage paraît prendre un sens indépendamment du mariage, et ce dernier acquiert probablement un sens nouveau pour ses plus jeunes adeptes.

En France, si le mariage "direct " a été la situation la plus fréquente pour les hommes des cohortes d'âge 45-49 ans et 4044 ans et pour les femmes des cohortes 45-49 ans, 40-44 ans et 35-39 ans, la cohabitation prénuptiale a constitué ensuite, dans un premier temps, une étape intermédiaire jusqu'au mariage. Les hommes et les femmes âgés de 30-34 ans à l'enquête se sont en effet mariés directement dans 21 pour cent et 35 pour cent des cas, et après avoir connu l'expérience d'une cohabitation hors mariage dans 39 pour cent et 32 pour cent des cas. Parmi les hommes et les femmes dont l'âge se situait entre 25 et 29 ans au milieu des années 1990, 10 pour cent des hommes et 18 pour cent des femmes ont déclaré s'être mariés sans avoir vécu au préalable avec leur partenaire, 19 pour cent des hommes et 30 pour cent des femmes ont officialisé leur union après une période de cohabitation hors mariage de moins de trois années, 10 pour cent des hommes et 14 pour cent des femmes ont eu un enfant dans les trois années de vie conjugale hors mariage, et 8 pour cent des hommes et 3 pour cent des femmes se sont séparés de leur premier partenaire. $\mathrm{Si}$, dans les cohortes les plus anciennes, la hausse de la cohabitation hors mariage a permis une certaine prise de distance entre la nuptialité et la conjugalité, dans les cohortes les plus récentes, la fécondité semble également se délier progressivement de la nuptialité.

En Italie, en revanche, la nuptialité est toujours dans la plupart des cas étroitement associée à la conjugalité et à la fécondité. De plus, les rares unions commencées hors mariage qui s'officialisent le font dans l'année de la mise en union.

En Suisse, si le mariage constitue toujours une étape importante du processus de conjugalité, dès la cohorte d'âge 40-44 ans pour les hommes et 35-39 ans pour les femmes, une période de cohabitation hors mariage le précède le plus souvent. La nuptialité se dissocie progressivement de la conjugalité, même si la grande majorité des premières unions s'achève toujours par un 
mariage. Seulement 7 pour cent des hommes de 25-29 ans et 4 pour cent des femmes du même âge se sont en effet séparés avant un mariage ou une naissance. Enfin, contrairement à ce qu'on observe en Suède mais aussi en France, où le nombre des unions non maritales fécondes augmente au fil des cohortes, les unions hors mariage restent la plupart du temps infécondes au sein de la Confédération helvétique, la fécondité restant étroitement associée à la nuptialité.

Parallèlement à la hausse du nombre des "unions sans papiers " en France, en Suède, en Suisse et, dans une proportion plus faible, en Italie, une hausse des désunions, variable selon les pays, s'observe dans les générations les plus récentes. Les unions hors mariage paraissent ainsi plus fragiles d'une cohorte à l'autre, et les individus insatisfaits de leur relation conjugale sont souvent plus disposés à interrompre leur première expérience conjugale avant de fonder une famille.

De manière différente et plus ou moins marquée selon les pays, la chronologie des événements familiaux s'est modifiée et la cohabitation hors mariage paraît constituer la première étape de ces nouvelles trajectoires. On peut s'interroger sur ce qui peut influencer le devenir de ces unions commencées hors mariage. Existe-t-il, par exemple, des prédispositions familiales ou contextuelles au devenir de la première union hors lien officiel ? Certains individus seraient-ils plus prompts à passer devant l'officier d'état civil, tandis que d'autres seraient plus enclins à terminer leur première expérience de vie de couple avant de connaître notamment une expérience de parentalité, ou seraient plus disposés à prolonger ce type de situation conjugale?

Pour mesurer le rôle des facteurs familiaux, contextuels et individuels, trois modèles semi-paramétriques ont été construits pour chaque sexe et pour chaque pays. Le premier s'intéresse à la survie dans l'état de cohabitant non marié, quel que soit le type de sortie (mariage ou séparation). Le deuxième modèle a été appliqué à la sortie par un mariage. Le troisième modèle testé s'intéresse au phénomène de la séparation. Dans ce cas, le mariage est considéré comme un phénomène entrant en concurrence (risque concurrent).

Les caractéristiques prises en compte sont: 1) le lieu de résidence principal pendant l'enfance; 2) la pratique religieuse (régulière, occasionnelle, nulle), de préférence à l'appartenance religieuse, car elle semble plus propre à influencer les comportements (Maréchal, 1997); 3) le divorce éventuel des parents avant le quinzième anniversaire, situation à laquelle pourraient être 
associées des attitudes différenciées face à une situation conjugale traditionnelle (Furstenberg et Teitler, 1994; Hullen, 1998; Wolfinger, 1999). Comme la première union s'inscrit le plus souvent dans une période d'âge marquée par de grands changements individuels, trois variables reflétant la situation au moment de l'union ont également été prises en compte : 4) le niveau de formation (primaire, secondaire, tertiaire) (Dechter, 1992); 5) le statut d'activité (en formation ou en recherche d'emploi, actif[ve], sans activité rémunérée) (Bracher et Santow, 1997); 6) l'éventuelle grossesse (Charton, 1999). Enfin, 7) l'âge au premier rapport sexuel (avant 16 ans, 16-17 ans, 18-19 ans, 20-23 ans, 24 ans et sans réponse) et 8) l'âge à la mise en première union (moins de 20 ans, 20-22 ans, 23-25 ans, 26-29 ans, 30 ans et plus) ont aussi été pris en compte, d'une part, pour saisir l'évolution d'une "distanciation " entre sexualité et conjugalité et, d'autre part, pour situer l'expérience de la première conjugalité dans le cycle de vie des individus (Thomson et Colella, 1992).

\section{TERMINER UNE PÉRIODE DE COHABITATION NON MARITALE}

\section{Une cohabitation hors mariage plus longue dans les générations les plus récentes}

Dans l'ensemble, près de huit premières unions observées chez les femmes et les hommes se sont achevées, soit par un mariage, soit par une séparation. Parmi les unions terminées, la durée de survie en état de cohabitant non marié est influencée par l'appartenance à la cohorte d'âge (voir le tableau B1 et le tableau B2, en annexe). Par rapport à la cohorte d'âge 45-49 ans en France et en Suisse, et à la cohorte d'âge 43 ans en Suède, l'union non maritale est en effet plus longue dans les cohortes les plus récentes ${ }^{1}:$ les risques relatifs tirés du modèle de survie sont significativement inférieurs à l'unité tant chez les femmes (compris entre 0,5 et 0,8 ) que chez les hommes (compris entre $0,4$ et 0,7$)$. En Italie, les hommes et les femmes âgés de moins de 45 ans ayant opté pour une union hors mariage paraissent, par contre, terminer plus rapidement leur union consensuelle comparativement à leurs aînés (âgés de 45-49 ans).

\section{Une cohabitation hors mariage plus courte pour une mise en couple avant 20 ans}

L'âge au moment de la mise en ménage joue également un rôle dans la conclusion de l'union non maritale. On constate en effet 
que le rythme de sortie est plus rapide pour les unions conclues avant 20 ans, par rapport à celles conclues plus tardivement. Cette tendance se confirme, comme nous le verrons plus loin, dans tous les pays, quel que soit le mode de sortie de la période de cohabitation (par un mariage ou une séparation).

\section{Un effet de l'âge des hommes au premier rapport sexuel sur la durée de l'union hors mariage}

L'âge au premier rapport sexuel semble avoir une influence sur le devenir de l'union non maritale pour les hommes en France et en Italie. Dans ces pays, les hommes qui ont déclaré avoir eu leur première relation sexuelle avant l'âge de 16 ans ont un risque statistique plus élevé d'achever leur union conjugale, comparativement à ceux qui ont eu une première expérience sexuelle plus tard.

Une réduction de la durée en cohabitation hors mariage pour une pratique religieuse régulière

La pratique religieuse a un effet important sur le devenir de l'union non maritale en augmentant fortement la probabilité de sortir de ce type d'union, en Suède et en Suisse, principalement par une officialisation de l'union. Cette variable parait, par contre, ne pas avoir d'effet significatif sur le devenir d'une union hors mariage en Italie, probablement parce que la plupart des personnes adoptant ce type d'union se déclarent non pratiquantes.

\section{Un effet variable du niveau de formation sur la durée de la cohabitation hors mariage}

Un niveau de formation tertiaire à la mise en union hors mariage augmente en France (significatif pour les hommes) et en Suède (pour les femmes) le risque statistique d'interrompre relativement vite ce type d'union. En Italie (pour les hommes) et en Suisse (pour les femmes), un niveau de formation élevé conduit, en revanche, plus fréquemment à prolonger une expérience conjugale hors mariage.

Il existe ainsi différentes caractéristiques sociodémographiques ayant une influence sur le devenir d'une première union commencée hors mariage. Il convient maintenant d'observer si l'interruption d'une union hors mariage se produit suite à une séparation ou à un mariage. 


\section{SE MARIER OU SE SÉPARER POUR CLORE UNE PREMIÈRE UNION NON MARITALE}

Une officialisation moins rapide et une séparation plus fréquente dans les générations les plus récentes

Les modèles considérant la survie de l'union non maritale face au mariage et face à la séparation montrent que si la cohorte d'âge d'appartenance paraît exercer un effet sur l'officialisation de l'union (sauf en Italie), elle a, selon les pays et le sexe des cohabitants, une influence variable sur la séparation (tableaux B1 et B2, en annexe).

Les hommes et les femmes des cohortes d'âge les plus récentes officialisent moins rapidement leur union que leurs aînés en France, en Suède et en Suisse. En Suède, c'est dans les cohortes d'âge de moins de 38 ans qu'on paraît, par ailleurs, le plus enclin à se séparer de son premier partenaire (la probabilité de se séparer oscille entre 1,5 et 2,5, comparativement aux 43 ans); il en va de même en France pour les hommes et les femmes de 20-24 ans (risque relatif respectif : 2,7 et 2,6, comparativement aux 45-49 ans), et en Suisse pour les hommes du même âge (risque relatif : 2,7 ).

\section{Se marier ou se séparer plus fréquemment} lors d'une mise en couple avant 20 ans

Les hommes et les femmes qui n'ont pas encore fêté leur vingtième anniversaire au moment de leur première mise en union se marient et se séparent plus rapidement que ceux et celles qui sont entrés en union à un âge plus avancé. Ainsi, les personnes qui ont expérimenté une cohabitation non maritale à un jeune âge prolongent moins souvent cette première expérience conjugale. Autrement dit, vivre une première union consensuelle à l'adolescence (avant 20 ans) conduit plus rapidement à en modifier les formes, par un mariage ou par une rupture.

\section{Influence de la zone de résidence durant l'enfance sur le devenir de l'union hors mariage}

En Italie, les personnes ayant commencé leur première union hors des liens du mariage ont une probabilité plus élevée d'officialiser leur union lorsqu'elles résidaient en zone rurale durant leur enfance, que celles qui ont grandi dans une commune plus importante. Cette tendance se retrouve également pour les hommes en Suisse. Dans ce pays, les femmes qui ont vécu leur 
enfance à l'étranger ont, par ailleurs, une probabilité significativement plus élevée (risque relatif : 1,3) de convoler que celles qui ont vécu en Suisse en zone rurale. Cette situation peut refléter les comportements conjugaux dominants des pays d'origine de ces femmes. La majorité des femmes étrangères sont, en effet, originaires d'Italie, d'Espagne et du Portugal; dans des proportions plus faibles, elles viennent de Turquie et d'ex-Yougoslavie (Wanner, 2001), c'est-à-dire de pays où la cohabitation hors mariage est un phénomène marginal.

En Suède, les hommes et les femmes qui ont résidé durant l'enfance dans une commune de plus de 10000 habitants ont une probabilité plus élevée de terminer leur première union consensuelle par une séparation que ceux qui ont vécu en zone rurale. Le lieu de vie et, plus généralement, des attaches sociales probablement plus denses semblent avoir une influence sur le mode de vie conjugal.

\section{Les non-pratiquants " résistent " mieux au mariage en Suède et en Suisse}

En Suède et en Suisse, la pratique régulière d'une religion a un effet positif sur l'officialisation de l'union. Le rôle de la pratique religieuse comme frein à la séparation n'est par contre pas démontré de manière significative. En Italie, la pratique religieuse n'influence pas le devenir de l'union hors mariage, probablement parce que la plupart des personnes en cohabitation se déclarent le plus souvent sans religion.

\section{Une officialisation de l'union plus rapide pour les femmes de formation supérieure en Suède, et en inactivité professionnelle en Italie}

En Suisse, les femmes de niveau de formation secondaire et tertiaire à leur mise en union convolent moins rapidement que les femmes de formation primaire. En Suède, en revanche, les femmes les plus diplômées (niveau d'études tertiaire) ont une probabilité plus élevée de terminer leur première union consensuelle par un mariage (risque relatif : 1,72). En France et en Italie, le niveau d'études des femmes n'a pas d'effet significatif sur le devenir de l'union. Un niveau d'études plus élevé prolonge, par contre, pour les hommes en Italie une cohabitation hors mariage, alors qu'en France un niveau d'études secondaire augmente la probabilité de se marier et un diplôme supérieur celle de se séparer (comparativement aux moins diplômés). 
En Italie, les femmes qui ont vécu leur première expérience de vie de couple hors mariage et qui n'exerçaient pas d'activité professionnelle rémunérée au moment de cette mise en couple terminent plus fréquemment leur union par un mariage (risque relatif : 1,72), tandis qu'en France elles se séparent moins fréquemment de leur conjoint que celles ayant un emploi rémunéré à la mise en couple (risque relatif : 0,42 ). En Suède et en Suisse, les femmes en formation ou à la recherche d'un emploi à leur mise en couple se séparent plus fréquemment de leur partenaire que les salariées (risque relatif respectif : 1,47 et 1,76). Les hommes en France qui n'exerçaient pas un emploi au moment de leur mise en couple prolongent une cohabitation hors mariage.

Il existe donc des différences, variables selon les pays, dans la manière de vivre sa première expérience de couple selon son niveau de formation et sa situation professionnelle. Ces observations peuvent vraisemblablement être rattachées aux représentations associées au couple et au mariage en fonction du pays et de la catégorie socioprofessionnelle des personnes.

\section{Une officialisation de l'union accélérée à l'annonce d'une grossesse en Suisse}

L'annonce d'une naissance a un impact fortement positif sur l'officialisation de l'union des couples vivant hors mariage, mais uniquement en Suisse. La probabilité de se marier est en effet respectivement, pour les hommes et les femmes, multipliée par 2,9 et 2,2 par rapport aux couples où la femme n'est pas enceinte. Une future naissance n'a en outre pas d'effet significatif sur le devenir de l'union consensuelle en France, en Italie et en Suède.

\section{CONCLUSION}

La cohabitation hors mariage s'est rapidement répandue au fil des générations, en Suède, puis en France et en Suisse. et elle concerne aujourd'hui quelques couples en Italie. La durée de la cohabitation hors mariage varie, par ailleurs, d'un couple à l'autre en fonction de facteurs socioculturels liés au processus de l'union cohabitale (Charton, 2003, 2006). Il a été observé que les facteurs qui agissent sur le choix du type d'union (maritale ou hors mariage) sont aussi ceux qui agissent sur le rythme de légalisation de l'union hors mariage, voire sur le rythme de rup- 
ture. Parmi ces facteurs, la cohorte d'âge et l'âge à la première mise en union ont une influence particulièrement importante, notamment dans les pays où la cohabitation hors mariage est fréquente. Les générations les plus récentes prolongent aussi une vie de couple hors mariage, tandis que les femmes et les hommes qui se sont "mis en couple " avant 20 ans terminent plus rapidement leur première union consensuelle.

Si un projet de mariage n'est plus indispensable pour vivre en couple en France, en Suède et en Suisse, la cohabitation reste toutefois encore dans ce dernier pays le plus souvent une étape de courte durée avant un probable mariage, notamment pour la formation d'une famille. La majorité des premières cohabitations aboutissent en effet le plus souvent au mariage, et l'annonce d'une grossesse augmente de manière significative une officialisation de l'union. Le mariage paraît donc "légitimer " l'annonce d'une naissance ${ }^{2}$. Le maintien, en Suisse, d'un modèle "traditionnel " dans lequel la cohabitation hors mariage reste inféconde et précède un mariage "quasi incontournable " peut être relié notamment à la difficulté de concilier vie professionnelle et vie familiale que ressentent les couples où les deux conjoints travaillent ${ }^{3}$. Cette difficulté favorise, en effet, le maintien d'un système dans lequel la femme renonce le plus souvent à son activité professionnelle pour s'occuper de ses enfants, tandis que le mari travaille à l'extérieur du foyer. Le mariage se présente alors comme le " ciment " de ce contrat familial.

En France, le devenir des unions consensuelles paraît corrélé plus spécifiquement au niveau de formation et au statut d'activité professionnelle des personnes. Les hommes de niveau de formation supérieur ont, par exemple, un risque statistique plus élevé de rompre leur première union hors mariage que les hommes de plus faible niveau de formation, peut-être parce que leur relation de couple ne constitue pas, du moins au début de leur vie d'adulte, une priorité de leur existence. Il est possible en effet, en raison d'un certain investissement scolaire, qu'ils préfèrent dans un premier temps faire "leurs preuves " ailleurs, notamment dans une activité professionnelle. Dans cet ordre d'idées, le fait que les femmes n'exerçant pas d'activité professionnelle au moment de leur mise en couple se séparent moins fréquemment de leur premier conjoint que leurs consœurs en emploi pourrait également être interprété comme un " choix " de "s'investir " en priorité dans une vie de couple. Ces observations reflètent également une répartition traditionnelle des rôles entre femmes et hommes au sein du couple. En Italie, les personnes 
qui optent pour une cohabitation hors mariage restent en nombre marginal. Parmi celles qui adoptent ce type de comportement " novateur ", le statut d'activité paraît également avoir une influence, puisqu'une inactivité professionnelle "encourage " une officialisation de l'union.

En Suède, où la cohabitation hors mariage est un phénomène largement répandu, le mariage, mais aussi la vie en couple, semblent se distancier de contraintes économiques. Le mariage comme garant d'une certaine sécurité matérielle, mais aussi comme événement privilégié aux dépens d'une activité professionnelle, paraît dépassé dans une certaine mesure. Ce sont les femmes du niveau de formation le plus élevé au moment de leur mise en union qui se marient, en effet, le plus rapidement, tandis que les femmes en recherche d'emploi ou en formation ont une probabilité plus importante de se séparer de leur conjoint que leurs consœurs en activité.

En définitive, la décision de "légitimer " une union hors mariage semble toujours, en France, en Italie et en Suisse, liée à certains aspects traditionnels, notamment à un rapport non égalitaire entre les sexes. En Suède, en revanche, le mariage et la vie de couple paraissent s'éloigner plus nettement de ce type de représentation. Le couple semble s'inscrire plus fréquemment dans la durée, voire dans le mariage, lorsque les individus se sont "stabilisés " professionnellement. Le couple se présente alors comme " élément constructif " d'une existence, au même titre vraisemblablement que d'autres expériences individuelles.

\section{NOTES}

1 Sauf pour les hommes de 20 à 24 ans en Suisse, et pour les hommes en Suède (risques statistiques non significatifs).

2 La prévalence de cette situation a, plus spécifiquement, été appréhendée en Suisse par la proportion élevée de premières naissances survenant dans les six mois qui suivent le mariage (Charton, 1999).

3 Notamment en raison de la capacité d'accueil insuffisante des crèches, de la difficulté du travail à temps partiel, des jongleries des horaires scolaires.

\section{RÉFÉRENCES BIBLIOGRAPHIQUES}

BECKER, G. S. 1960. "An economic analysis of fertility", dans R EASTERLIN, éd. Demographic and Economic Change in Developed Countries. Princeton, Princeton University Press : 209-231. 
BECKER, G. S. 1981. A Treatise on the Family, Harvard, Harvard University Press, 320 p.

BLOSSFELD, H. P., A. MAMERLE et K. U. MAYER. 1989. Event History Analysis: Statistical Theory and Application in the Social Sciences. Hillsdale (NJ), L. Erlbaum Associates Publishers, 295 p.

BLOSSFELD, H. P., et G. ROHWER. 1995. Techniques of Event History Modeling. New Approches to Causal Analysis. Mahwah (NJ), L. Erlbaum Associates Publishers, 294 p.

BRACHER, M., et G. SANTOW. 1997. Economic Independence and Union Formation in Sweden. Stockholm University, Stockholm Research Reports in Demography, 116, 41 p.

CHARTON, L. 1999. "De l'union légalisée à la naissance légalisée : évolution du lien entre mariage et premier enfant en Suisse ", Cahiers québécois de démographie, 28, 1-2 : 151-172.

CHARTON, L. 2003. Calendriers familiaux et rapport au temps. La diversification des comportements et des parcours familiaux en Europe. Strasbourg, Université Marc Bloch, thèse de doctorat en sciences sociales, $546 \mathrm{p}$.

CHARTON, L. 2006. Familles contemporaines et temporalités, Paris, L'Harmattan, Collection Logiques sociales, à paraître.

CHARTON, L., et J. DUCHÊNE. 2002. "Évolution des comportements familiaux et nouvelles structures des ménages. Une étude comparative entre sept pays européens ", dans Vivre plus longtemps, avoir moins d'enfants, quelles implications? [actes du] Colloque international de Byblos-Jbeil (Liban, 10-13 octobre 2000), AIDELF, no 10. Paris, PUF : 309-322.

CLIQUET, R. L. 1991. La deuxième transition démographique : réalité ou fiction ? Strasbourg, éd. du Conseil de l'Europe, Études démographiques, 23, $113 \mathrm{p}$.

COX, D. 1972. "Regression models and life tables ", Journal of the Royal Statistical Society, Series BJ, 34 : 187-220.

DECHTER, A. R. 1992. The Effect of Women's Economic Independence on Union Dissolution. University of Wisconsin, Center for Demography and Ecology, CDE Working Paper 92-28, 5 p.

DORMONT, B., et S. DUFOUR-KIPPELEN. 2000. "Insertion professionnelle et autonomie résidentielle : le cas des jeunes peu diplômés ", Économie et statistique, 337-338: 97-120.

EASTERLIN, R. 1976. "The conflict between aspirations and resources ", Population and Development Review, 2, 3-4 : 417-425.

ECE (Economic Commission For Europe). 1989. Outline of the Framework for Fertility and Family Survey in The Early 1990s in the ECE Region. Genève, ONU, $116 \mathrm{p}$. 
EKERT-JAFFE, O., et A. SOLAZ. 2001. "Unemployement and formation of the family in France ", The Journal of Socio-economics, 30 : 75-98.

ENGLAND, P., et G. FARKAS. 1986. Households, Employment and Gender. A Social, Economic and Demographic View. New York, Aldine De Gruyter, 237 p.

FURSTENBERG, F. F., et J. O. TEITLER. 1994. "Reconsidering the effect of marital disruption: What happens to children of divorce in early adulthood? ", Journal of Family Issues, 15 : 173-190.

HOFFMANN-NOWOTNY, H. J. 1988. "Structural and cultural determinants of Europe's second demographic transition ", Bevolking in Gezin, $1: 73-89$.

HOFFMANN-NOWOTNY, H. J., et B. FUX. 1991. "Tendances démographiques actuelles en Europe ", Actes du Séminaire sur les tendances démographiques actuelles et modes de vie en Europe, Strasbourg 1820 septembre, Strasbourg, éd. du Conseil de l'Europe : 31-97.

HÖHN, C. 1997. "Bevölkerungsentwicklung und demographische Herausforderung ", dans S. HRADIL et S. IMMERFALL, éd. Die westeuropäischen Gesellschaften im Vergleich. Leske/Budrich, Opladen : 71-96.

HULLEN, G. 1998. "Scheidungskinder-oder: die Transmission des Scheidungsrisikos ", Zeitschrift für Bevölkerungswissenschaft, 23, 1 : 19-38.

KAUFMANN, J.-C. 1994. "Nuptialité ou conjugalité ? Critique d'un indicateur et état des évolutions conjugales en Europe ", Archives européennes de sociologie, 35, 1 : 3-20.

KLIJZING, E., et M. MACURA. 1996. Cohabitation and Extra-marital Chilbearing: Early FFS Evidence. Genève, PAU/UN, 24 p., miméo.

LESTHAEGHE, R. 1983. "A century of demographic and cultural change in western Europe. An exploration of underlying dimensions ", Population and Development Review, 9, 3 : 411-435.

MARÉCHAL, M. 1997. Cycle de vie et milieu social selon l'Enquête Famille de 1990, INSEE Résultats, Démographie-Société, 62-63, 219 p.

OPPENHEIMER, V. K. 1988. "A theory of marriage timing ", American Journal of Sociology, 94 : 563-591.

OPPENHEIMER, V. K. 1997. "Women's employment and the gain to marriage. The specialization and trading model ", Annual Review of Sociology, 23 : 431-453.

PAVLIK, Z. 1989. Demographic Revolution as a Part of a Global Revolution of Modern Times of the Concept of Development. Dubrovnik, InterUniversity Centre of Post-graduate Studies, séminaire " Demographic Trends and Population Policy ", 38 p., mimeo. 
PINNELLI, A., H. J. HOFFMANN-NOWOTNY et B. FUX. 2001. Fécondité et nouveaux types de ménages et de formation de la famille en Europe, Strasbourg, éd. du Conseil de l'Europe, Études démographiques, 35, $193 \mathrm{p}$.

PONTHIEUX, S. 1997. "Débuter dans la vie active au milieu des années 90 : des conditions qui se dégradent ", Économie et Statistique, 304305, 4/5 : 37-51.

RINDFUSS, R., et A. VANDEN HEUVEL. 1990. "Cohabitation: A precursor to marriage or an alternative to being single? ", Population and Development Review, 16, 4 : 703-726.

ROUSSEL, L. 1978. "La cohabitation juvénile en France ", Population, $1: 15-42$.

ROUSSEL, L. 1989. La famille incertaine. Paris, O. Jacob, 283 p.

ROUSSEL, L. 1992. "La famille en Europe occidentale : divergences et convergences ", Population, 1 : 133-152.

SINGLY, F. de. 1992. La famille : transformations récentes. Problèmes politiques et sociaux, 685, $61 \mathrm{p}$.

THOMSON, E., et U. COLELLA. 1992. "Cohabitation and marital stability: Quality or commitment? ", Journal of Marriage and The Family, $54: 259-267$.

TROST, J. 1975. "Married and unmarried cohabitation: The case of Sweden, with some comparisons ", Journal of Marriage and the Family, 37 : 677-682.

VAN DE KAA, D. J. 1987. "Europe's second demographic transition ", Population Bulletin, 42 : 1-59.

VILLENEUVE-GOKALP, C. 1990. "Du mariage aux unions sans papiers : histoire récente des transformations conjugales ", Population, 45, 2 : 265-296.

WANNER, P. 2001. Immigration en Suisse. Situation et conséquences démographiques. Neuchâtel, OFS, 63 p.

WOLFINGER, N. H. 1999. "Trends in the intergenerational transmission of divorce ", Demography, 36, 3 : 415-420. 


\section{Annexe}

TABLEAU A. Forme de la première union et devenir de l'union hors mariage sur une période de trois années, femmes et hommes ayant entre 30 ans et 50 ans aux enquêtes FFS (proportions pondérées)

\begin{tabular}{|c|c|c|c|c|c|c|c|c|c|c|}
\hline \multirow{2}{*}{$\begin{array}{l}\text { Groupes d'âge } \\
\text { aux enquêtes }\end{array}$} & \multicolumn{5}{|c|}{ Femmes } & \multicolumn{5}{|c|}{ Hommes } \\
\hline & $45-49$ & $40-44$ & $35-39$ & $30-34$ & $25-29$ & $45-49$ & $40-44$ & $35-39$ & $30-34$ & $25-29$ \\
\hline \multicolumn{11}{|l|}{ France } \\
\hline Mariage direct & 73,4 & 67,7 & 51,7 & 35,4 & 18,1 & 65,6 & 52,7 & 38,5 & 20,8 & 10,2 \\
\hline Prélude & 13,8 & 19,7 & 28,5 & 32,4 & 29,7 & 22,6 & 29,8 & 32,7 & 39,1 & 19,4 \\
\hline Union hors mariage féconde & 4,4 & 5,0 & 7,8 & 14,0 & 13,5 & 1,8 & 5,9 & 11,5 & 12,7 & 10,5 \\
\hline Union stable & 3,0 & 2,7 & 3,5 & 4,9 & 12,0 & 2,9 & 2,7 & 6,0 & 8,3 & 12,0 \\
\hline Union éphémère & 0,0 & 0,3 & 0,2 & 1,5 & 1,1 & 0,3 & 1,7 & 0,5 & 1,0 & 2,2 \\
\hline Union rompue & 0,5 & 0,3 & 1,0 & 1,5 & 1,9 & 0,9 & 0,7 & 0,1 & 1,1 & 5,9 \\
\hline Sans union & 4,6 & 4,2 & 7,1 & 8,7 & 16,4 & 5,9 & 6,5 & 9,5 & 13,9 & 31,2 \\
\hline Union " tronquée " à l'enquête & 0,3 & 0,1 & 0,2 & 1,6 & 7,3 & 0,0 & 0,0 & 1,2 & 3,1 & 8,6 \\
\hline \multicolumn{11}{|l|}{ Italie } \\
\hline Mariage direct & 91,9 & 88,2 & 83,6 & 75,1 & 48,4 & 87,7 & 82,4 & 73,4 & 56,8 & 15,8 \\
\hline Prélude & 2,2 & 3,5 & 4,5 & 6,4 & 3,6 & 1,4 & 5,7 & 6,8 & 4,9 & 3,5 \\
\hline Union hors mariage féconde & 0,6 & 0,9 & 1,3 & 1,4 & 0,6 & 0,6 & 0,5 & 1,6 & 0,4 & 0,0 \\
\hline Union stable & 0,4 & 0,2 & 0,7 & 0,4 & 0,4 & 0,0 & 1,0 & 1,1 & 0,2 & 1,4 \\
\hline Union éphémère & 0,0 & 0,0 & 0,1 & 0,0 & 0,2 & 0,0 & 0,0 & 1,9 & 1,0 & 2,2 \\
\hline Union rompue & 0,0 & 0,1 & 0,1 & 0,0 & 0,3 & 0,0 & 1,0 & 0,0 & 2,0 & 1,1 \\
\hline Sans union & 4,7 & 7,1 & 9,7 & 16,1 & 45,1 & 10,3 & 8,6 & 14,6 & 34,3 & 75,4 \\
\hline Union " tronquée " à l'enquête & 0,2 & 0,0 & 0,0 & 0,6 & 1,4 & 0,0 & 0,8 & 0,6 & 0,4 & 0,6 \\
\hline
\end{tabular}


Suède

\begin{tabular}{|c|c|c|c|c|c|c|c|c|c|c|}
\hline \multicolumn{2}{|l|}{ Mariage direct } & 18,4 & 9,6 & 7,4 & 7,3 & & 11,6 & & 5,5 & 4,6 \\
\hline \multicolumn{2}{|l|}{ Prélude } & 55,3 & 54,9 & 45,5 & 34,0 & & 57,7 & & 39,9 & 22,7 \\
\hline \multicolumn{2}{|l|}{ Union hors mariage féconde } & 17,4 & 22,1 & 24,7 & 22,0 & & 13,8 & & 20,2 & 19,8 \\
\hline \multicolumn{2}{|l|}{ Union stable } & 4,5 & 6,5 & 13,0 & 13,4 & & 9,3 & & 16,3 & 14,2 \\
\hline \multicolumn{2}{|l|}{ Union éphémère } & 0,2 & 0,8 & 1,0 & 1,8 & & 0,9 & & 1,1 & 3,7 \\
\hline \multicolumn{2}{|l|}{ Union rompue } & 0,7 & 0,8 & 2,1 & 4,3 & & 0,6 & & 3,4 & 6,5 \\
\hline \multicolumn{2}{|l|}{ Sans union } & 3,3 & 4,7 & 4,4 & 10,2 & & 5,8 & & 10,7 & 18,8 \\
\hline \multicolumn{2}{|l|}{ Union " tronquée " à l'enquête } & 0,2 & 0,6 & 1,9 & 7,0 & & 0,3 & & 2,9 & 9,7 \\
\hline \multicolumn{11}{|l|}{ Suisse } \\
\hline Mariage direct & 67,5 & 45,7 & 32,1 & 22,4 & 18,8 & 61,3 & 39,0 & 29,6 & 21,3 & 13,7 \\
\hline Prélude & 22,9 & 36,9 & 47,9 & 50,8 & 35,7 & 24,4 & 44,4 & 46,5 & 41,5 & 22,5 \\
\hline Union hors mariage féconde & 2,5 & 4,0 & 5,2 & 2,5 & 2,0 & 2,6 & 3,4 & 5,7 & 2,7 & 2,4 \\
\hline Union stable & 2,1 & 5,1 & 5,9 & 8,3 & 9,7 & 3,3 & 6,1 & 6,0 & 10,4 & 7,3 \\
\hline Union éphémère & 0,5 & 0,3 & 0,1 & 0,9 & 1,2 & 0,4 & 0,4 & 0,5 & 0,6 & 1,8 \\
\hline Union rompue & 0,4 & 1,5 & 0,9 & 2,7 & 2,3 & 0,4 & 1,0 & 2,5 & 3,9 & 4,7 \\
\hline Sans union & 4,1 & 5,7 & 6,8 & 8,7 & 18,7 & 7,6 & 5,7 & 6,8 & 13,1 & 33,9 \\
\hline Union " tronquée " à l'enquête & 0,0 & 0,8 & 1,1 & 3,7 & 11,6 & 0,0 & 0,0 & 2,4 & 6,5 & 13,7 \\
\hline
\end{tabular}

Adapté partiellement de la classification proposée par C. Villeneuve-Gokalp (1990).

Mariage direct: mariage non précédé d'une union consensuelle. Prélude : union suivie d'un mariage au cours des trois ans qui suivent la mise en union. Union hors mariage féconde : union suivie d'une naissance au cours des trois premières années, sans mariage. Union stable : union suivie d'aucun mariage ni naissance au cours des trois ans qui suivent la mise en union. Union éphémère : union rompue au cours des 12 premiers mois. Sans union: union rompue au bout de 12 à 36 mois de cohabitation. Sans union : individu n'ayant pas encore vécu une première union au moment de l'enquête. Union "tronquée " à l'enquête : union en cours tronquée par l'enquête (non observée sur une durée de trois années). 
TABLEAU B1. Facteurs influençant le devenir de l'union non maritale au cours de la première expérience de vie de couple, hommes et femmes, France et Italie (modèles semi-paramétriques, risques relatifs)

\begin{tabular}{|c|c|c|c|c|c|c|c|c|c|c|c|c|}
\hline \multirow[b]{4}{*}{ Facteurs } & \multicolumn{6}{|c|}{ France } & \multicolumn{6}{|c|}{ Italie } \\
\hline & \multicolumn{2}{|c|}{ Toutes fins d'union } & \multicolumn{2}{|c|}{ Mariage } & \multicolumn{2}{|c|}{ Séparation } & \multicolumn{2}{|c|}{ Toutes fins d'union } & \multicolumn{2}{|c|}{ Mariage } & \multicolumn{2}{|c|}{ Séparation } \\
\hline & $\mathrm{H}$ & $\mathrm{F}$ & $\mathrm{H}$ & $\mathrm{F}$ & $\mathrm{H}$ & $\mathrm{F}$ & $\mathrm{H}$ & $\mathrm{F}$ & $\mathrm{H}$ & $\mathrm{F}$ & $\mathrm{H}$ & F \\
\hline & $67,5 \%$ & $63,6 \%$ & $40,9 \%$ & $40,0 \%$ & $26,6 \%$ & $23,6 \%$ & $86,3 \%$ & $50,0 \%$ & $40,0 \%$ & $38,9 \%$ & $46,3 \%$ & $11,1 \%$ \\
\hline \multicolumn{13}{|c|}{ Groupes d'âge aux enquêtes FFS } \\
\hline $20-24$ ans & $0,61 *$ & $0,58 * * *$ & $0,11 * * *$ & $0,23 * * *$ & $2,72 * *$ & $2,56 * * *$ & $4,48(2)^{* *}$ & $2,94 * *$ & $2,54(2)$ & 1,69 & $5,13(2)$ & $41,89 * * *$ \\
\hline $25-29$ ans & $0,37 * * *$ & $0,54 * * *$ & $0,16 * * *$ & $0,35 * * *$ & 1,40 & 1,43 & & 1,23 & & 0,96 & & 2,14 \\
\hline $30-34$ ans & $0,50 * * *$ & $0,66^{* *}$ & $0,39 * * *$ & $0,50 * * *$ & 0,99 & 1,22 & 1,54 & 1,57 & 1,00 & 1,53 & 2,29 & 1,34 \\
\hline $35-39$ ans & $0,63 * *$ & 0,84 & $0,48 * * *$ & 0,79 & 1,33 & 1,01 & $3,03 *$ & 1,04 & 1,50 & 0,78 & 2,29 & 2,14 \\
\hline $40-44$ ans & 0,91 & 0,92 & 0,91 & 0,91 & 0,99 & 0,94 & (3) & 0,76 & (3) & 0,73 & (3) & 0,78 \\
\hline $45-49$ ans & Réf. & Réf. & Réf. & Réf. & Réf. & Réf. & Réf. & Réf. & Réf. & Réf. & Réf. & Réf. \\
\hline \multicolumn{13}{|c|}{ Lieu de résidence durant l'enfance } \\
\hline Moins de 2000 habitants & & & & & & & Réf. & Réf. & Réf. & Réf. & Réf. & Réf. \\
\hline 2000 à 9999 hab. & & & & & & & 0,42 & $0,53 *$ & $0,04 *$ & $0,47 *$ & 2,69 & 0,81 \\
\hline 10000 à 99999 hab. & & & & & & & 0,93 & 0,86 & $0,15 *$ & 0,72 & 2,71 & 1,48 \\
\hline$\geq 100000$ hab. et étrange & & & & & & & 1,22 & 0,72 & 1,05 & 0,70 & 2,57 & 0,66 \\
\hline \multicolumn{13}{|l|}{ Pratique religieuse } \\
\hline Pratiquant régulier & & & & & & & Réf. & Réf. & Réf. & Réf. & Réf. & Réf. \\
\hline Pratiquant occasionnel & & & & & & & 0,57 & 0,97 & 0,33 & 1,03 & 1,21 & 1,10 \\
\hline Non pratiquant & & & & & & & 1,11 & 0,83 & 0,32 & 0,79 & 4,05 & 1,38 \\
\hline \multicolumn{13}{|l|}{ Parents divorcés? } \\
\hline Non & 1,14 & 1,02 & 1,27 & 1,19 & 1,01 & 0,81 & 1,69 & 1,00 & 9,24 & 1,45 & 0,40 & $0,32 * *$ \\
\hline Oui & Réf. & Réf. & Réf. & Réf. & Réf. & Réf. & Réf. & Réf. & Réf. & Réf. & Réf. & Réf. \\
\hline
\end{tabular}


Niveau de formation atteint (1)

\begin{tabular}{|c|c|c|c|c|c|c|c|c|c|c|c|c|}
\hline Primaire & Réf. & Réf. & Réf. & Réf. & Réf. & Réf. & Réf. & Réf. & Réf. & Réf. & Réf. & Réf. \\
\hline Secondaire & $1,38 * *$ & 1,01 & $1,41 * *$ & 1,01 & 1,34 & 1,02 & $0,17 * * *$ & 1,29 & $0,05 * * *$ & 1,22 & 1,78 & 2,47 \\
\hline Tertiaire & 1,28 & 1,02 & 0,93 & 0,87 & $1,81 * *$ & 1,31 & $0,28 *$ & 0,79 & $0,03 * * *$ & 0,60 & 7,17 & 2,39 \\
\hline \multicolumn{13}{|l|}{ Statut professionnel (1) } \\
\hline En activité occupée & Réf. & Réf. & Réf. & Réf. & Réf. & Réf. & Réf. & Réf. & Réf. & Réf. & Réf. & Réf. \\
\hline Autre & 0,93 & 0,86 & $0,77 * *$ & 1,17 & 1,17 & $0,42 * * *$ & 1,25 & $1,55 *$ & 2,79 & $1,72 *$ & 0,66 & 0,62 \\
\hline $\begin{array}{l}\text { Au chômage ou en } \\
\text { formation (femmes) }\end{array}$ & & 1,02 & & 1,03 & & 1,00 & & 1,20 & & 1,03 & & 1,19 \\
\hline \multicolumn{13}{|c|}{ Âge au premier rapport sexuel } \\
\hline Moins de 16 ans & Réf. & Réf. & Réf. & Réf. & Réf. & Réf. & Réf. & Réf. & Réf. & Réf. & Réf. & Réf. \\
\hline $16-17$ ans & $0,77 *$ & 1,04 & $0,75 *$ & 1,06 & 0,83 & 0,99 & 1,01 & 0,91 & 1,46 & 1,28 & 0,64 & $0,38 *$ \\
\hline $18-19$ ans & 0,84 & 1,03 & 0,83 & 1,02 & 0,84 & 1,03 & $0,15 * * *$ & 0,79 & 0,37 & 1,12 & $0,05 * * *$ & $0,26 * *$ \\
\hline $20-23$ ans & 0,80 & 1,04 & 0,95 & 1,18 & $0,52 *$ & 0,80 & 0,89 & 0,88 & 1,86 & 1,43 & 0,30 & $0,17 *$ \\
\hline 24 ans et plus & 0,97 & 0,70 & 1,18 & 0,89 & 0,49 & 0,38 & $0,08 *$ & 1,08 & 0,45 & 1,39 & $0,00 * *$ & 0,59 \\
\hline Sans réponse & $0,67 *$ & 0,74 & 0,69 & 0,82 & 0,57 & 0,61 & 1,16 & 0,89 & 2,12 & 1,40 & 1,20 & 0,00 \\
\hline \multicolumn{13}{|c|}{ Enceinte ou partenaire enceinte ? (1) } \\
\hline Non & Réf. & Réf. & Réf. & Réf. & Réf. & Réf. & & Réf. & & Réf. & & Réf. \\
\hline Oui & 0,83 & 0,97 & 1,06 & 0,88 & 0,50 & 1,15 & & 1,28 & & 1,62 & & 0,17 \\
\hline \multicolumn{13}{|l|}{ Âge à l'entrée en union } \\
\hline Moins de 20 ans & Réf. & Réf. & Réf. & Réf. & Réf. & Réf. & Réf. & Réf. & Réf. & Réf. & Réf. & Réf. \\
\hline $20-22$ ans & $0,42 * * *$ & $0,47 * * *$ & $0,46 * * *$ & $0,48 * * *$ & $0,41 * * *$ & $0,44 * * *$ & $0,15 * *$ & $0,39 * * *$ & $0,04 * *$ & $0,38 * * *$ & 0,45 & $0,27 *$ \\
\hline $23-25$ ans & $0,18 * * *$ & $0,27 * * *$ & $0,21 * * *$ & $0,25 * * *$ & $0,16 * * *$ & $0,30 * * *$ & $0,02 * * *$ & $0,21 * * *$ & $0,00 * * *$ & $0,18 * * *$ & $0,08 * *$ & $0,29 *$ \\
\hline $26-29$ ans & $0,10 * * *$ & $0,18 * * *$ & $0,10 * * *$ & $0,17 * * *$ & $0,09 * * *$ & $0,18 * * *$ & $0,02 * * *$ & $0,15 * * *$ & $0,00 * * *$ & $0,14^{* * *}$ & $0,01 * * *$ & 0,19 ** \\
\hline 30 ans et plus & $0,04 * * *$ & $0,10 * * *$ & $0,05 * * *$ & $0,11 * * *$ & $0,03 * * *$ & $0,10 * * *$ & $0,00 * * *$ & $0,06 * * *$ & $0,00 * * *$ & $0,05 * *$ & $0,00 * * *$ & $0,10 * * * *$ \\
\hline
\end{tabular}

(1) Au moment de l'union. (2) Groupe d'âge 20-29 ans. (3) Groupe d'âge non pris en compte dans le modèle.

Seuils de signification: * 5 (pour 100); ** 1 (pour 100) et *** 1 (pour 1000).

Lecture : les hommes en France qui ont atteint un niveau de formation supérieure au moment de leur mise en union ont une probabilité, à chaque durée d'union, 1,81 fois plus élevée (au risque statistique de $1 \%$ ) que les hommes de niveau de formation primaire (référence) de terminer leur union consensuelle par une séparation, toutes choses égales par ailleurs. 
TABLEAU B2. Facteurs influençant le devenir de l'union non maritale au cours de la première expérience de vie de couple, hommes et femmes, Suède et Suisse (modèles semi-paramétriques, risques relatifs)

\begin{tabular}{|c|c|c|c|c|c|c|c|c|c|c|c|c|}
\hline \multirow[b]{3}{*}{ Facteurs } & \multicolumn{6}{|c|}{ Suède } & \multicolumn{6}{|c|}{ Suisse } \\
\hline & \multicolumn{2}{|c|}{ Toutes fins d'union } & \multicolumn{2}{|c|}{ Mariage } & \multicolumn{2}{|c|}{ Séparation } & \multicolumn{2}{|c|}{ Toutes fins d'union } & \multicolumn{2}{|c|}{ Mariage } & \multicolumn{2}{|c|}{ Séparation } \\
\hline & $\begin{array}{c}\mathrm{H} \\
81,0 \%\end{array}$ & $\begin{array}{c}\mathrm{F} \\
78,6 \%\end{array}$ & $\begin{array}{c}\mathrm{H} \\
34,4 \%\end{array}$ & $\begin{array}{c}F \\
46,0 \%\end{array}$ & $\begin{array}{c}\mathrm{H} \\
46,7 \%\end{array}$ & $\begin{array}{c}\mathrm{F} \\
32,6 \%\end{array}$ & $\begin{array}{c}\mathrm{H} \\
87,5 \%\end{array}$ & $\begin{array}{c}F \\
89,4 \%\end{array}$ & $\begin{array}{c}\mathrm{H} \\
65,7 \%\end{array}$ & $\begin{array}{c}F \\
68,4 \%\end{array}$ & $\begin{array}{c}\mathrm{H} \\
21,9 \%\end{array}$ & $\begin{array}{c}F \\
21,0 \%\end{array}$ \\
\hline \multicolumn{13}{|c|}{ Groupes d'âge aux enquêtes FFS } \\
\hline $20-24$ ans & & 0,91 & & $0,36 * * *$ & & $2,46 * * *$ & $1,68 *$ & 0,86 & 1,18 & 0,92 & $2,65 *$ & 1,10 \\
\hline $25-29$ ans & 1,06 & $0,84 *$ & $0,65 * * *$ & $0,55 * * *$ & $2,00 * * *$ & $1,82 * * *$ & 0,91 & $0,72 * *$ & 0,83 & 0,83 & 1,16 & 1,25 \\
\hline $30-34$ ans & 0,94 & $0,76 * * *$ & $0,71 * *$ & $0,55 * * *$ & $1,55^{* *}$ & $1,52 * * *$ & 0,88 & $0,81 *$ & 0,86 & 0,87 & 0,97 & 1,09 \\
\hline 35-39 ans & & $0,73 * * *$ & & $0,64 * * *$ & & 1,06 & $0,71 * *$ & $0,73 * *$ & $0,75 *$ & $0,75 * *$ & 0,64 & 0,80 \\
\hline $40-44$ ans & Réf. & Réf. & Réf. & Réf. & Réf. & Réf. & 0,80 & $0,81 *$ & 0,84 & 0,89 & 0,73 & 1,15 \\
\hline $45-49$ ans & & & & & & & Réf. & Réf. & Réf. & Réf. & Réf. & Réf. \\
\hline \multicolumn{13}{|c|}{ Lieu de résidence durant l'enfance } \\
\hline Moins de 2000 habitants & Réf. & Réf. & Réf. & Réf. & Réf. & Réf. & Réf. & Réf. & Réf. & Réf. & Réf. & Réf. \\
\hline 2000 à 9999 hab. & 1,04 & 1,06 & 0,90 & 1,13 & 1,26 & 0,97 & $0,75 * *$ & 0,90 & $0,74 * *$ & 0,89 & 0,81 & 0,86 \\
\hline 10000 à 99999 hab. & $1,18 *$ & $1,19 * *$ & 1,11 & $1,17 *$ & $1,31 *$ & $1,22 *$ & $0,79 *$ & 1,11 & $0,69 * *$ & 1,05 & 1,21 & 0,90 \\
\hline$\geq 100000$ hab. et étrang. & $1,38 * * *$ & $1,39 * * *$ & 1,12 & 1,19 & $1,83 * * *$ & $1,70 * * *$ & $0,70 * *$ & 0,93 & $0,68 * *$ & 0,95 & 0,82 & 0,99 \\
\hline Étranger (en Suisse seult) & & & & & & & 0,81 & $1,35 * *$ & 0,84 & $1,27 * *$ & 0,74 & 1,05 \\
\hline \multicolumn{13}{|l|}{ Pratique religieuse } \\
\hline Pratiquant régulier & Réf. & Réf. & Réf. & Réf. & Réf. & Réf. & Réf. & Réf. & Réf. & Réf. & Réf. & Réf. \\
\hline Pratiquant occasionnel & $0,60 * *$ & $0,82 *$ & $0,48 * * *$ & $0,78 *$ & 0,87 & 0,90 & $0,67 * *$ & $0,84 *$ & $0,60 * * *$ & $0,80 * *$ & 1,18 & 0,65 \\
\hline Non pratiquant & $0,46 * * *$ & $0,68 * * *$ & $0,37 * * *$ & $0,54 * * *$ & 0,68 & 1,01 & $0,60 * * *$ & $0,49 * * *$ & $0,48^{* * *}$ & $0,56 * * *$ & 1,52 & 0,79 \\
\hline \multicolumn{13}{|l|}{ Parents divorcés? } \\
\hline Non & 0,92 & $0,86 *$ & 0,92 & 0,91 & 0,93 & $0,81 *$ & 1,04 & $1,30 * *$ & 1,02 & 1,13 & 1,11 & 0,81 \\
\hline Oui & Réf. & Réf. & Réf. & Réf. & Réf. & Réf. & Réf. & Réf. & Réf. & Réf. & Réf. & Réf. \\
\hline
\end{tabular}


Niveau de formation atteint (1)

\begin{tabular}{|c|c|c|c|c|c|c|c|c|c|c|c|c|}
\hline Primaire & Réf. & Réf. & Réf. & Réf. & Réf. & Réf. & Réf. & Réf. & Réf. & Réf. & Réf. & Réf. \\
\hline Secondaire & 1,25 & 1,23 & 1,17 & 1,48 & 1,38 & 0,81 & 0,93 & $0,78 * *$ & 1,02 & $0,77 * *$ & 0,79 & 0,79 \\
\hline Tertiaire & 1,22 & $1,43 *$ & 1,26 & $1,72 *$ & 1,18 & 0,93 & 0,92 & $0,68 * *$ & 0,96 & $0,72 * *$ & 0,90 & 0,90 \\
\hline \multicolumn{13}{|c|}{ Statut professionnel (1) } \\
\hline En activité occupée & Réf. & Réf. & Réf. & Réf. & Réf. & Réf. & Réf. & Réf. & Réf. & Réf. & Réf. & Réf. \\
\hline Autre & 1,05 & 1,34 & 0,81 & 1,47 & 1,30 & 1,22 & 1,14 & 1,04 & 1,00 & 1,14 & $1,56 *$ & 1,44 \\
\hline $\begin{array}{l}\text { Au chômage ou en } \\
\text { formation (femmes) }\end{array}$ & & $1,27 * *$ & & 1,13 & & $1,47^{* * *}$ & & $0,68 * *$ & & 0,91 & & $1,76 * * *$ \\
\hline \multicolumn{13}{|c|}{ Âge au premier rapport sexuel } \\
\hline Moins de 16 ans & & & & & & & Réf. & Réf. & Réf. & Réf. & Réf. & Réf. \\
\hline $16-17$ ans & & & & & & & 0,84 & 1,11 & 0,89 & 1,00 & 0,76 & 0,86 \\
\hline $18-19$ ans & & & & & & & 0,87 & 0,97 & 0,92 & 0,93 & 0,79 & 0,94 \\
\hline $20-23$ ans & & & & & & & 0,86 & 1,06 & 0,92 & 0,95 & 0,76 & 0,74 \\
\hline 24 ans et plus & & & & & & & 0,75 & 1,08 & 0,90 & 1,02 & $0,43 *$ & 1,02 \\
\hline Sans réponse & & & & & & & 0,98 & 1,13 & 1,19 & 1,01 & 0,37 & 0,76 \\
\hline \multicolumn{13}{|c|}{ Enceinte ou partenaire enceinte ?(1) } \\
\hline Non & Réf. & Réf. & Réf. & Réf. & Réf. & Réf. & Réf. & Réf. & Réf. & Réf. & Réf. & Réf. \\
\hline Oui & 1,13 & 0,98 & 1,35 & 1,11 & 0,79 & 0,79 & $2,32 * * *$ & 2,68 *** & $2,87^{* * *}$ & $2,24 * * *$ & 0,55 & 0,54 \\
\hline \multicolumn{13}{|c|}{ Âge à l'entrée en union } \\
\hline Moins de 20 ans & Réf. & Réf. & Réf. & Réf. & Réf. & Réf. & Réf. & Réf. & Réf. & Réf. & Réf. & Réf. \\
\hline $20-22$ ans & $0,62 * * *$ & $0,52 * * *$ & 0,82 & $0,61 * * *$ & $0,47^{* * *}$ & $0,41^{* * *}$ & $0,49 * * *$ & $0,44^{* * *}$ & $0,63 *$ & $0,39 * * *$ & $0,33 * * *$ & $0,31 * * *$ \\
\hline $23-25$ ans & $0,36 * *$ & $0,31 * * *$ & $0,50 * * *$ & $0,32 * * *$ & $0,26 * * *$ & $0,30 * * *$ & $0,24 * * *$ & $0,20 * * *$ & $0,32 * * *$ & $0,16 * * *$ & $0,16 * * *$ & $0,10 * * *$ \\
\hline $26-29$ ans & $0,19 * * *$ & $0,19 * * *$ & $0,27 * * *$ & $0,21 * * *$ & $0,13 * * *$ & $0,16 * * *$ & $0,10 * * *$ & $0,11^{* * *}$ & $0,15 * * *$ & $0,09 * * *$ & $0,04 * * *$ & $0,05 * * *$ \\
\hline 30 ans et plus & $0,12 * * *$ & $0,12 * * *$ & $0,15 * * *$ & $0,13 * * *$ & $0,08 * * *$ & $0,12 * * *$ & $0,04 * * *$ & $0,03 * * *$ & $0,05 * * *$ & $0,03 * * *$ & $0,02 * *$ & $0,02 * * *$ \\
\hline
\end{tabular}

(1) Au moment de l'union.

Seuils de signification : * 5 (pour 100); ** 1 (pour 100) et *** 1 (pour 1000).

Lecture : en Suède, les femmes qui ont atteint un niveau supérieur de formation au moment de leur mise en union ont, à chaque durée d'union, une probabilité 1,72 fois plus élevée (au risque statistique de $5 \%$ ). 


\begin{abstract}
Laurence CHARTON

THE EVOLUTION OF THE FIRST UNION BEGUN OUTSIDE MARRIAGE: A COMPARATIVE ANALYSIS OF FRANCE, ITALY, SWEDEN AND SWITZERLAND BASED ON FERTILITY AND FAMILY SURVEYS OF THE 1990S

Today, in Europe, more and more couples are entering into a first conjugal experience outside marriage. Various questions are being raised about the evolution of these unions. Are they more precarious than marital unions? Are they temporary episodes of conjugal life? Do they lead to marriage? Are they a new, parallel form of union outside marriage? Are there different forms of cohabitation outside marriage in different countries? And finally, what role do the family context and social environment play in the evolution of the union? This article tries to answer some of these questions based on data gathered in the 1990s by Fertility and Family Surveys (FFS) conducted in France, Italy, Sweden and Switzerland, countries that represent the variety of conjugal and fertility situations in western Europe. Life history analysis methods were used to investigate the time intervals and individual and contextual characteristics involved.
\end{abstract}

